\title{
Reflexiones sobre las perspectivas teóricas de la (T.E) Tecnología Educativa
}

\author{
Reflections about the theoretical perspectives of educational \\ technologies
}

Reflexões sobre as perspectivas teóricas da Tecnologia Educacional (T.E)

José Antonio Correa Padilla

Magister en diseño de entornos virtuales de aprendizaje Corporación Universitaria del Caribe CECAR- Virtual jose.correap@cecar.edu.co

\section{Resumen}

En el transcurso del tiempo la tecnología educativa ha tenido una metamorfosis en cuanto a las perspectivas y conceptualización, sin estas perder la esencia de su objetivo, en este artículo se muestran éstas transformaciones y su incidencia en el ámbito educativo.

Palabras claves: Tecnología educativa, educación, pedagogía.

\section{Summary}

Over the course of time educational technology has had a metamorphosis in terms of perspectives and conceptualization, without losing the essence of its objective, this article shows these transformations and their impact on the educational field.

Keywords: Educational technology, education, pedagogy.

\section{Resumo}

Ao longo do tempo, a tecnologia educacional teve uma metamorfose em termos de perspectivas e conceituação, sem perder a essência de seu 
objetivo, este artigo mostra essas transformações e seu impacto no campo educacional.

Palavras-chave: Tecnologia educacional, educação, pedagogia.

La ciencia desde sus inicios busca un poco de verdad en cada paso que da, desde sus investigaciones, verificaciones $\mathrm{e}$ hipótesis. Algunas veces encuentra verdades que desbancan a las anteriores. En otras encuentra que cada teoría o perspectiva ilumina un poco más acerca de un mismo tema complementando el panorama comprensivo del mismo. De igual forma, la tecnología va desarrollando soluciones gradualmente, teniendo en cuenta las necesidades y/o el objeto de su intervención.

Tomando como base estas últimas premisas, se considera que las perspectivas teóricas de la tecnología educativa son complementarias. De hecho, cada perspectiva aporta un elemento adicional de gran valor al conocimiento general acerca del tema y, efectivamente, en los diseños tecnológicos educativos actuales se logran percibir elementos de cada una de las diferentes épocas: sea desde su concepción, uso o filosofía subyacente.

Por esta razón, es importante comprender los puntos comunes que todas las perspectivas tienen para consolidar el objeto de estudio general de la tecnología educativa. Al respecto, todas se refieren al diseño, utilización y desarrollo de artefactos y procesos relacionados con el aprendizaje. Todas tienen además, una concepción positiva del aprendizaje ya que lo asumen como una actividad que produce bienestar y desarrollo, enfatizando en la necesidad de una buena actuación en el proceso educativo, es decir contemplan el panorama ético y efectivo en todos los procesos relacionados con la tecnología educativa.

De esta manera, es importante hacer evidente que estamos en una época dentro de la historia misma del conocimiento y el mundo, donde en cada momento de la vida estamos haciendo uso consciente $\mathrm{o}$ inconscientementede actividades que tienen sus referentes $y$ bases en otras anteriores, por ello, es de vital importancia encontrar los puntos comunes y las conexiones básicas sobre las cuales tomar decisiones efectivas. Lo anterior implica hacer referencia al conectivismo, teoría que cada vez toma más fuerza como forma de comprensión del fenómeno del aprendizaje en la era digital.

Siendo consecuente con la premisa anterior, es necesario percibir la tecnología educativa como una disciplina que se alimenta de los desarrollos en todas las perspectivas a través del tiempo. Por ello y 
pensando/sintiendo desde el campo emotivo- conductual, se considera de gran utilidad la perspectiva cognitiva-mediacional como necesaria en los contextos educativos, puesto que tiene en cuenta para definir el objeto y aplicaciones de la tecnología educativa, no solo aquellos aspectos que rodean al individuo que aprende, sino aquellos de su interior, con el fin de diseñar y desarrollar recursos de todo tipo que posibiliten el desarrollo efectivo de una tarea cognitiva compleja de índole superior, en este caso, el aprendizaje.

Unido a lo anterior, esta perspectiva cumple criterios de amplia rigurosidad científica como la riqueza en la descripción, efectividad en la explicación y capacidad de réplica en sus resultados (herencia del conductismo). Aunque también cumple con criterios contextuales y subjetivos en los cuales tiene en cuenta las variables que afectan al sujeto desde su historia personal $y$ el contexto donde se desenvuelve (precursor del campo reflexivo-cultural), explicando el universo simbólico creado desde la interacción entre las estructuras cognitivas de la persona y el mundo que lo rodea.

Es necesario entonces aclarar que las perspectivas teóricas no son puras, por lo tanto, en ciertas coyunturas se apoyan unas a otras. Sin embargo, a través del tiempo las perspectivas teóricas tienen un acercamiento más profundo con ciertos criterios; inclusive hoy en día los seres humanos obtienen el conocimiento desde otros canales que ni si quiera eran contemplados en el pasado, como las diversas redes sociales mediante la conectividad. Derivado de esto se considera que las definiciones van evolucionando respecto a su planteamiento original.

Sabemos bien que en el transcurso del tiempo la tecnología educativa ha tenido una metamorfosis en cuanto a las perspectivas y conceptualización, sin estas perder la esencia de su objetivo. Cabe entonces aclarar que se complementan, aunque en un comienzo tenía un enfoque diferente; no obstante, con el tiempo se fue acortando la brecha que existe entre la tecnología y la educación.

Las etapas rápidas del desarrollo tecnológico han permitido a la sociedad el acceso a la información y conocimiento con mayor productividad. La integración de varias ciencias refleja la adquisición de conocimientos y nuevas experiencias en el aprendizaje.

Las perspectivas de la tecnología educativa desarrollan de manera significativa el trabajo en equipo, donde se mezclan varias ramas de la ciencia y la educación, generando así mejores resultados en sus acciones, profundizando en sus saberes y transmitiendo conocimientos, facilitando en su mayoría la comunicación entre las personas. 
Es necesario considerar entonces que las perspectivas teóricas aportan un punto de vista real y necesario que de alguna manera se van integrando con las demás.

El proceso para considerarla como una disciplina en sí misma, ha sido lento, pues siempre se ha encontrado al margen de los aportes realizados desde las otras disciplinas sin destacar como tal.

Como planteó el autor Pere Marquès (1999), en la década de los 60 se articula la Tecnología Educativa como campo de estudio diferenciado, incorporando conocimientos desde las Ciencias Sociales.

En las últimas décadas y con el avance abismal de las TIC, se ha hecho más evidente la necesidad de seguir profundizando en la tecnología educativa como disciplina en sí, asumiéndola no solo como una simple congruencia de aportes.

Como todo campo de estudio, hay que tomarlo como tal, con sus aportes y deficiencias, sin pensar que la misma solucionará problemas de aprendizaje por sí misma como arte de magia.

La concepción sobre la tecnología educativa va acompañada además, de procesos de cambios de paradigmas en la educación. Si bien antes era el docente el centro, ahora pasa a ser el alumno, así que esa postura de centrarse en la enseñanza de la información da paso al docente guía-mediador a que busque integrar de manera innovadora y acertada las TIC a sus clases.

Sin embargo, no es tan simple como parece, pues según una investigación realizada en los 90 en EE.UU se detectan diez puntos que intervienen en el uso efectivo de las TIC en la educación, como por ejemplo, el dinero; la infraestructura; la falta de visión del profesorado; falta de soporte técnico, entre otros (Luján y Salas, 2009. p.4).

En conclusión, se podría asegurar que aunque se ha avanzado en esta disciplina, queda mucho camino por recorrer porque es mucho más compleja y abarca más de lo que se podría pensar.

Después de realizar la lectura pertinente, no me atrevo a decir con total certeza que las perspectivas teóricas de la tecnología educativa son complementarias porque cada una surgió y se desarrolló como respuesta a una deficiencia mostrada por su predecesora, o como un movimiento que representaba soluciones a lagunas de las problemáticas inmersas en ellas. Sin embargo, lo que sí me queda muy claro es que no son opuestas, incluso cuando algunas intentan posicionarse en fundamentos y prácticas muy distintas a las de otras perspectivas. En últimas todas rescatan el factor tecnológico y la importancia del diseño como elementos destacados para lograr la eficiencia buscada en el proceso de enseñanza. 
Es claro entonces, que con el paso de los años se fueron agregando elementos primordiales, como el contacto con estímulos externos y la forma en que cada persona aprende (cognitivismo); ya no solo son importantes los instrumentos de aprendizaje programados (conductismo); si no que además es necesario tener en cuenta el entorno, la cultura y el medio social. Este proceso ha sido más que una contrariedad o complementación, es una evolución necesaria que se ha ido acoplando a las necesidades cambiantes tanto de la sociedad en general como de las oportunidades que la tecnología ofrece para facilitar la enseñanza.

En lo particular, el enfoque cognitivo es el más cercano a la visión de la tecnología educativa, porque difumina un poco la diferencia entre el tecnólogo diseñador de intervenciones y el profesor aplicador de las mismas. Hoy en día los profesores debemos ser orientadores, diseñadores, planeadores, gestores y facilitadores al mismo tiempo del conocimiento. Por otro lado, también se debe compartir con los estudiantes la perspectiva de un aprendizaje basado en experiencias y estímulos cotidianos de la vida real, para que sientan que lo que aprenden tiene una aplicación práctica en la vida.

Así mismo, el enfoque crítico reflexivo es fundamental dado que la enseñanza debe responder al medio en el que se encuentra inmerso el estudiante, su contexto particular y en ocasiones debe ser diseñado desde la reflexión de las necesidades específicas de ellos, para que asimilen desde una posición en la que la información como un elemento valioso $y$ enriquecedor.

Para concluir, se hace necesario añadir que, aunque el conductismo es un enfoque un poco hecho a un lado, todos los profesores tenemos actuaciones que en un determinado momento se compaginan con este enfoque, sobre todo cuando nos vemos inmersos en situaciones disciplinarias. En estos casos, el reto es ofrecerle al estudiante un escenario motivacional que lo impulse a encontrar su propio motor de impulso para el aprendizaje.

\section{Referencias}

Marqués, P. (1999). La tecnología educativa: conceptualización, líneas de investigación [Documento electrónico]. Recuperado en mayo 8 de 2017 https://ticsunermb.wordpress.c om/2008/04/08/la-tecnologiaeducativa-conceptualizacionlineas-de-investigacion-por-drpere-marques-graells-1999ultima-revision-30707/

Área, M (2011). Tic, identidad digital y educación. Cuatro reflexiones Reencuentro, 62, pp. 97-99. 
Cabero, J. (s.f). Líneas y tendencias de investigación en medios de enseñanza [Documento electrónico]. Recuperado en $\begin{array}{llll}\text { mayo } & 8 & \text { de } & 2017\end{array}$ de http://edutec.rediris.es/docu mentos/1991/4.htm

Darder. A (2014). Organización y gestión de la tutoría online para la dirección de proyectos de investigación. Tesis doctoral inédita. Universitat de les Illes Balears, Palma de Mallorca.

Gómez, J. (2013). Neurociencia Cognitiva y Educación. Chiclayo: Fondo Editorial FACHSE.

Olmedo, E. (s.f). Tecnología Educativa: conceptualización y fundamentación. Documento de clase.

Siemens G. (2013). Conectivismo: Una Teoría de Aprendizaje para la Era Digital. Recuperado en mayo 8 de 2017 desde http://www.comenius.cl/recurs os/virtual/minsal_v2/Modulo_1 /Recursos/Lectura/conectivism o_Siemens.pdf

Luján Ferrer, M y Salas Madriz, F. (2009) Enfoques teóricos y definiciones de la tecnología educativa en el siglo xx. Actualidades Investigativas en Educación, 9(2) ,1-29. 


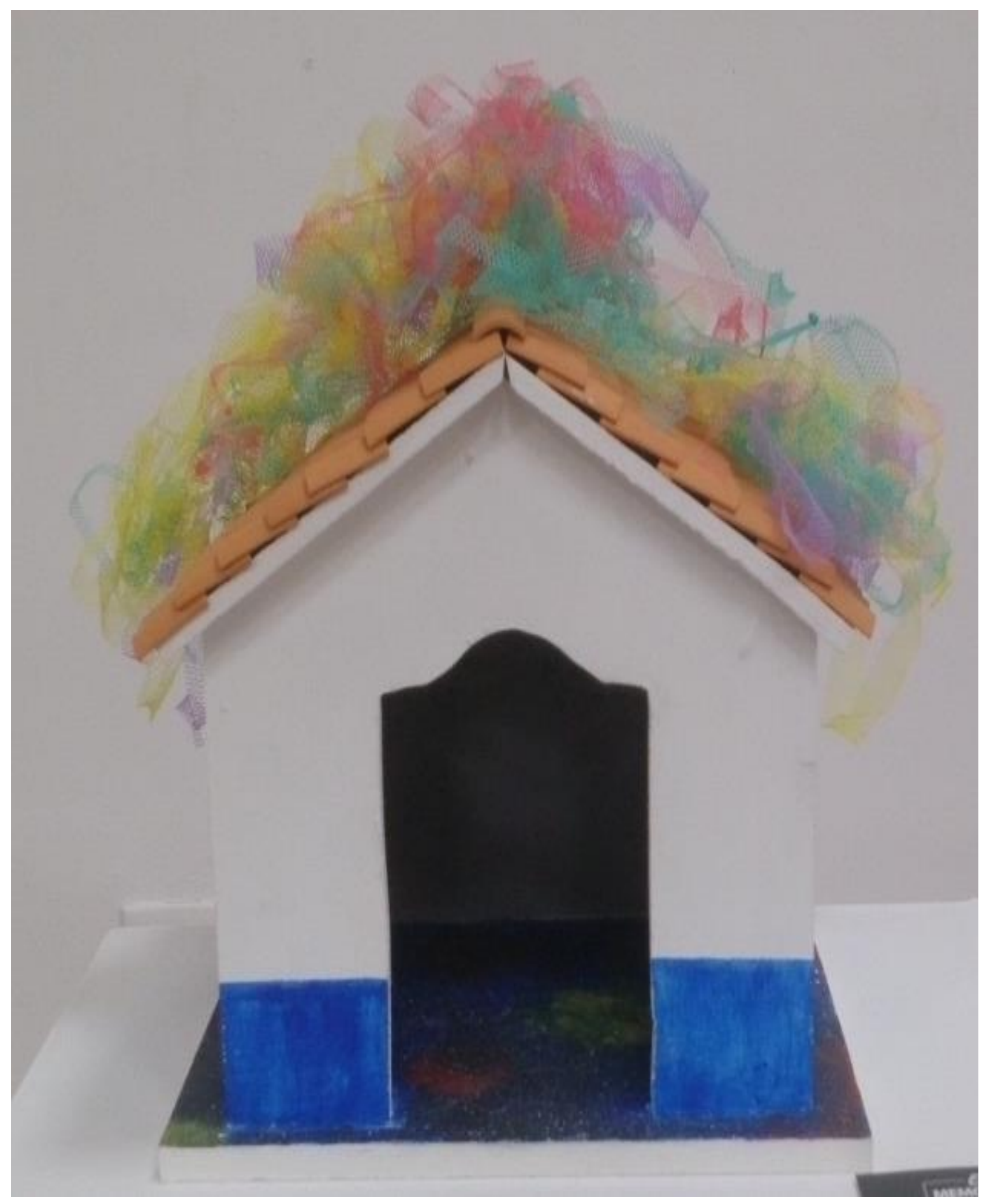

Obra: Alma infinita

Artista: Pablo Mosquera (Lovitz)

Técnica: Acrílico sobre maderas - telas

Territorio: Neiva 